\title{
Integrated in silico-in vitro characterization, identification and disruption of the intermolecular interaction between SH3 domain-containing protein kinases and human pituitary tumor-transforming gene 1
}

\author{
Yanan $\mathrm{Li}^{1, \star}$, Mengmeng Li², ${ }^{2,}$ Weijie Min ${ }^{1}$, Guosheng Han ${ }^{1}$, Laixing Wang ${ }^{1}$, Chao Chen ${ }^{1}, \mathrm{Zifu} \mathrm{Li}^{1}$, \\ Yuhui Zhang ${ }^{1}$, Jianmin Liu ${ }^{1}$ and Zhijian Yue ${ }^{1}$ \\ ${ }^{1}$ Department of Neurosurgery, Changhai Hospital, Second Military Medical University, Shanghai 200433, China \\ ${ }^{2}$ Department of Rheumatology and Immunology, Changzheng Hospital, Second Military Medical University, Shanghai 200003, \\ China
}

\begin{abstract}
The human pituitary tumor-transforming gene-1 (hPTTG1) has been found to be overexpressed in various cancers. Accumulated evidences implicate that some of protein kinases can specifically recognize two PXXP motifs at hPTTG1 C-terminus through their Src homology (SH3) domain and then phosphorylate the protein by their catalytic domain. Here, we integrate in silico analysis and in vitro assay to characterize the intermolecular interaction between the two hPTTG1 motif peptides ${ }^{161}$ LGPPSPVK $^{168}$ (M1P) and ${ }^{168} \mathrm{KMPSPPWE}^{175}$ (M2P) and the SH3 domains of Ser/ Thr-specific protein kinases MAP3K and PI3K. It is identified that the two peptides bind to MAP3K SH3 domain with a moderate affinity, but cannot form stable complexes with PI3K SH3 domain. Long time scale molecular dynamics (MD) simulations reveal that the M1P peptide can fold into a standard poly-proline II helix that is bound in the peptide-binding pocket of MAP3K SH3 domain, while the M2P peptide gradually moves out of the pocket during the simulations and finally forms a weak, transient encounter complex with the domain. All these suggest that the MAP3K M1P site is a potential interacting partner of MAP3K SH3 domain, which may mediate the intermolecular recognition between hPTTG1 and MAP3K.
\end{abstract}

Key words: Human pituitary tumor-transforming gene 1 - Protein kinase - SH3 domain - Peptide - Endocrinology

\section{Introduction}

Pituitary tumor-transforming gene 1 (PTTG1) was first isolated from rat pituitary tumor cells in 1997 (Pei and Melmed 1997), and subsequently identified as a common oncogenic protein in vertebrate that is overexpressed in a variety of tumors, including those from pituitary, breast, thyroid, ovar-

\footnotetext{
* These authors contributed equally to this work.

Correspondence to: Jianmin Liu, Department of Neurosurgery, Changhai Hospital, Second Military Medical University, Shanghai 200433, China

E-mail: liu118@vip.163.com

Zhijian Yue, Department of Neurosurgery, Changhai Hospital, Second Military Medical University, Shanghai 200433, China

E-mail: yuezj638@163.com
}

ian, uterine, colon and lung (Vlotides et al. 2007). Human PTTG1 (hPTTG1) is involved in multiple cellular pathways, including proliferation, DNA repair, transformation, angiogenesis induction, invasion and the induction of genetic instability, which affects tumor invasiveness and recurrence in several systems, functions as a securin during cell cycle progression, and inhibits premature sister chromatid separation (Salehi et al. 2008). In recent years, the hPTTG1 has been established as a promising prognostic marker and new therapeutic target for pituitary adenomas, specifically in hormone-secreting tumors, in which invasiveness correlates with high levels of expression (Zhang et al. 1999a; Tfelt-Hansen et al. 2006).

Chromatin immunoprecipitation-on-chip study revealed that hPTTG1 is a global transcription factor, which exerts its transcriptional activity by either directly binding to DNA or indirectly mediating various proteins such as PTTG1IP, 
PTTG1 binding factor, separase, p53, Sp1, MEK1, upstream stimulatory factor 1 and diverse shock proteins (Tong and Eigler 2009). In order to uncover the structural basis of hPTTG1 capable of interacting with so many biomolecular partners, Sánchez-Puig and co-workers employed biophysical characterizations to show that hPTTG1 is totally unstructured in solution and thus belongs to the family of natively unfolded proteins, which is devoid of tertiary and secondary structure except for a small amount of polyproline II (PPII) helix at its C-terminal region (Boelaert et al. 2004; Sánchez-Puig et al. 2005). Under physiological condition, the intermolecular interaction events of hPTTG1 with its diverse binding partners are regulated by a complicated combination of phosphorylation and dephosphorylation events at a variety of phosphorylatable sites (Tong and Eigler 2009). The intact hPTTG1 protein is composed of 202 amino acids, in which more than 30 residues are serine, threonine and tyrosine, implying that the protein is a primary target of protein kinases. For example, rat PTTG1 has a consensus phosphorylation site $\mathrm{X}(\mathrm{S} / \mathrm{T})$ $\mathrm{P}$ within the transactivation domain and is phosphorylated by Ser/Thr-specific protein kinase at Ser162 in vitro, which is critical for rat PTTG1 transactivation function (Pei 2000).

A proline-rich region in hPTTG1 C-terminus contains two PXXP motifs that are potential binding targets of protein kinases having a Src homology 3 (SH3) domain (Boelaert et al. 2004). Mutation or deletion of the hPTTG1 SH3-binding motifs totally abolished transforming activity and transactivating ability of the protein, suggesting that the motifs are required for hPTTG1 functions including transformation, bFGF induction and angiogenesis (Domínguez et al. 1998; Zhang et al. 1999b). Served as a multifunctional regulator, the hPTTG1 has been found to interact with various proteins and to participate in a variety of cell signaling pathways (Tong and Eigler 2009). A number of protein kinases are involved in these pathways, which regulate hPTTG1 functions by phosphorylating the protein and its interacting factors. Protein kinases can specifically recognize and phosphorylate hPTTG1 through three approaches: (i) Low-efficiency approach: the kinase directly catches the substrate by itself; (ii) Mediumefficiency approach: the kinase interacts with the substrate via a scaffolding protein which can bind the kinase and the substrate simultaneously; (iii) High-efficiency approach: the kinase recognizes the substrate using a regulatory module such as the $\mathrm{SH} 3$ domain. In this study, we only considered the third approach. Many kinases such as Src and Abl contain a noncatalytic $\mathrm{SH} 3$ domain that functions as regulatory module to determine kinase's substrate specificity by selectively targeting PXXP motif present in the substrate proteins. Previously, cellbased assays revealed that the hPTTG1 signaling pathway is essentially regulated by $\mathrm{SH} 3$-containing kinases MAP3K and PI3K (Pei 2000; Vlotides et al. 2006; Chen et al. 2013; Li et al. 2013). Thus, we assume that the two kinases may specifically recognize hPTTG1 SH3-binding motifs via their SH3 domain and then phosphorylates the hPTTG1 by their catalytic domain. In order to test the notion, we herein propose a synthetic strategy that integrates in silico analysis and in vitro assay to systematically characterize the intermolecular interaction between hPTTG1 SH3-binding motifs and the SH3 domain of MAP3K and PI3K. Based on the knowledge harvested from the analysis we also design two peptide mutants that may have potent capability to competitively disrupt the hPTTG1-kinase interaction. This work would help to elucidate the molecular mechanism and biological implication underlying hPTTG1 recognition and phosphorylation by protein kinases and to establish a synthetic protocol for the biological function inference and rational drug design of multifunctional proteins in tumor signaling networks.

\section{Materials and Methods}

Modeling complex structures of MAP3K and PI3K SH3 domains with hPTTG1 SH3-binding motifs

The hPTTG1 has two SH3-binding motifs ${ }^{163} \mathrm{PPSP}^{166}$ and ${ }^{170} \mathrm{PSPP}^{173}$ present in its C-terminal region (UniProt:

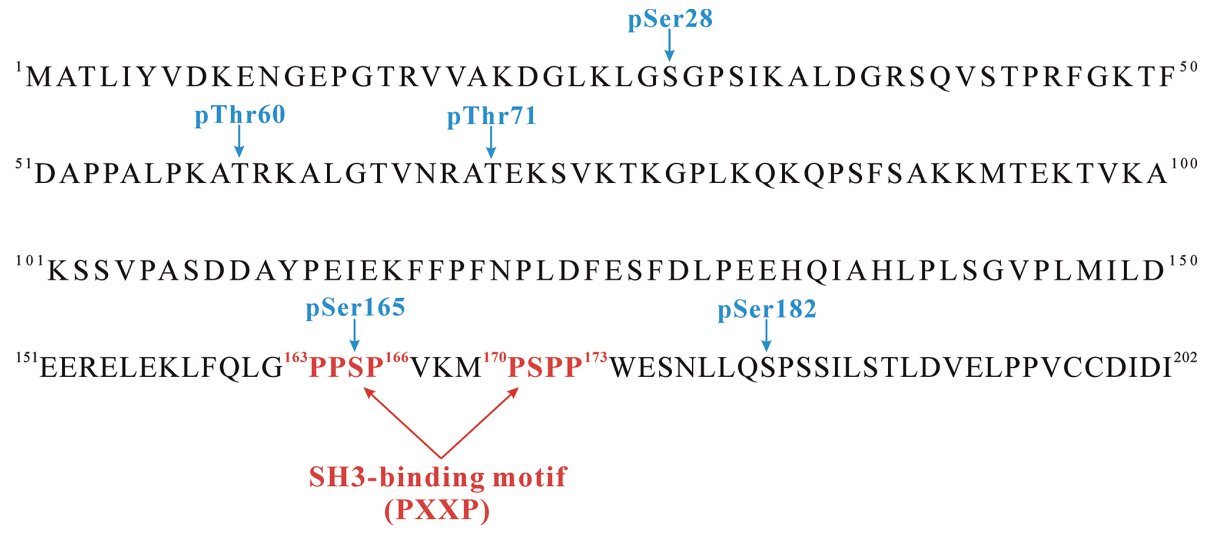

Figure 1. The hPTTG1 sequence (UniProt: O95997) as well as two SH3-binding motifs and five known phosphorylation sites in the sequence. 
O95997) (Unipro 2010). Here, the sequence information of hPTTG1 as well as the two SH3-binding motifs and five known phosphorylation sites (pSer28, pThr60, pThr71, pSer165 and pSer182) in the sequence are shown in Figure 1. Considering that the residues flanking to the motifs can also contribute to SH3-ligand binding affinity and specificity, each side of a motif was extended with two additional residues to define an 8 -mer sequence as the potential binding site of SH3 domain. Consequently, two extended octapeptides ${ }^{161}$ LGPPSPVK $^{168}$ (M1P) and ${ }^{168}$ KMPSP$\mathrm{PWE}^{175}$ (M2P) separately covering the two SH3-binding motifs were derived.

The complex crystal structure of PI3K SH3 domain with a 9-mer peptide KRPLPPLPS and the crystal structure of $a p o$ MAP3K SH3 domain were retrieved from the PDB database (Berman et al. 2000) with accession codes 3I5R and 2RF0, respectively. First, we modeled the complex structures of PI3K $\mathrm{SH} 3$ domain with M1P and M2P peptides. The procedure is as follows: (i) Removal of the most C-terminal Ser residue of the co-crystallizated nonapeptide, resulting in the complex of PI3K SH3 domain with octapeptide KRPLPPLP. (ii) Virtual mutagenesis of the octapeptide to M1P and M2P peptide by using a graph theory-based SCWRL algorithm (Krivov et al. 2009), resulting in the low-quality complex structures of PI3K SH3 domain with M1P and M2P. (iii) The structures were minimized with YASARA server (Krieger et al. 2002) to eliminate bad atomic contacts, resulting in the refined complex structures of PI3K SH3 domain with M1P and M2P.

Subsequently, we modeled the complex structures of MAP3K SH3 domain with M1P and M2P. Since the crystal structure of MAP3K SH3 domain is in apo state that has no co-crystallizated peptide ligand, we employed a computational strategy to graft the M1P and M2P peptides from the modeled PI3K SH3-peptide complexes to MAP3K SH3 domain. The strategy is as follows: (i) Superposition of MAP3K SH3 domain to PI3K SH3 domain in complex with M1P or M2P peptide, resulting in superposed PI3K SH3/ peptide/MAP3K SH3 system. (ii) Removal of the PI3K SH3 domain from the superposed system, resulting in artificial MAP3K SH3-peptide complex. (iii) The artificial MAP3K $\mathrm{SH} 3$-peptide complex was minimized with YASARA server (Krieger et al. 2002), resulting in the refined complex structure of MAP3K SH3 domain with peptide.

\section{Molecular dynamics simulations}

Molecular dynamics (MD) methodology has been widely used to investigate protein-peptide binding phenomena, such as self-binding peptides (Yang et al. 2015, 2016). Here, each of the domain-peptide complex structures modeled above was solvated in a rectangular box full of explicit TIP3P water molecules (Jorgensen et al. 1983) extended $10 \AA$ from the complex in $x, y$ and $z$ orientations. First, the complex was in turn relaxed by 500 cycles of steepest descent minimization and 3000 cycles of conjugate gradient minimization. Subsequently, 100-ns MD simulations with time step of $2.0 \mathrm{fs}$ were performed at a constant temperature of $300 \mathrm{~K}$ and a constant pressure of $1 \mathrm{~atm}$. The Particle Mesh Ewald (PME) method (Darden et al. 1993) was used to calculate the full electrostatic energy of a unit cell in a macroscopic lattice of repeating images, and SHAKE method (Ryckaert et al. 1977) was employed to constrain all covalent bonds involving hydrogen atoms.

During the last 10-ns stable simulations snapshots of the domain-peptide complex were saved every 2 ps, yielding a total of 500 snapshots, which were used for binding free energy calculations. Molecular mechanics/Poisson-Boltzmann surface area (MM/PBSA) method was applied to the calculations (Homeyer and Gohlke 2012), which derived the interaction energy $\Delta E_{\text {int }}$ between the domain and peptide and the desolvation free energy $\Delta G_{\mathrm{dslv}}$ upon the domainpeptide binding using molecular mechanics (MM) approach and implicit solvent model (PBSA), respectively. To consider the conformational flexibility of peptide ligand, the normal mode analysis (NMA) (Moritsugu et al. 2010) was employed to estimate entropy penalty $-T \Delta S$ upon the binding. Due to the high computational demand only 50 snapshots were used in the analysis (Hou et al. 2006). Frequencies of vibrational modes were computed at $300 \mathrm{~K}$ for the snapshots using a harmonic approximation of the energies. Consequently, the total binding free energy $\Delta G_{\text {total }}$ of peptide to domain can be obtained as:

$$
\Delta G_{\text {total }}=\Delta E_{\text {int }}+\Delta G_{\mathrm{dslv}}-T \Delta S
$$

Here, all dynamics simulations and energetics calculations were carried out using amber ff03 force field (Duan et al. 2003) implemented with the AMBER11 suite of programs (Case et al. 2005).

\section{Fluorescence spectroscopy assay}

Peptides were synthesized using standard 9-fluorenyl methoxycarbonyl (Fmoc) solid phase chemistry, which were then purified by RP-HPLC C18 columns and confirmed by mass spectrometry and amino acid analysis. A standard fluorescence spectroscopy protocol modified from previous studies (Pisabarro and Serrano 1996; Schweimer et al. 2002) was used to perform peptide binding assay. Briefly, the fluorescence emission spectra of fluorescently active residues around the peptide-binding pocket of $\mathrm{SH} 3$ domain were used to monitor change in their environment upon peptide binding. Fluorescence was measured by a PerkinElmer LS-55 fluorescence spectrophotometer at an excitation wavelength of $298 \mathrm{~nm}$ (slit width $2 \mathrm{~nm}$ ) and an emission wavelength of $345 \mathrm{~nm}$ (slit width $4 \mathrm{~nm}$ ). The SH3 concentration was kept 
at a constant $(10 \mu \mathrm{M})$ in solution $(10 \mathrm{mM} \mathrm{KPi}, \mathrm{pH} 7.0)$. Changes in fluorescence were measured upon titration of peptide solution $(6.5 \mathrm{mM})$. All experiments were performed at $25^{\circ} \mathrm{C}$. Peptide affinity $K_{\mathrm{d}}$ was obtained by fitting experimental data to the equation:

$$
F=\left[F_{0}+F_{\infty}\left([\text { pep }] / K_{\mathrm{d}}\right)\right] /\left[1+\left([\text { pep }] / K_{\mathrm{d}}\right)\right]
$$

where [pep] is the peptide concentration at each measurement point, $F$ is the measured $\mathrm{SH} 3$ fluorescence intensity at the given peptide concentration, $F_{0}$ is intensity in absence of peptide, and $F_{\infty}$ is the maximal fluorescence intensity of the $\mathrm{SH} 3$ when saturated with the peptide.

\section{Results and Discussion}

The dynamics stability and energetics analysis of domainpeptide complexes

The complex structures of MAP3K and PI3K SH3 domains with M1P and M2P peptides were computationally modeled using virtual mutagenesis and grafting strategy, resulting in four complex systems namely MAP3K SH3-M1P, MAP3K SH3-M2P, PI3K SH3-M1P and PI3K-M2P. In order to explore structural dynamics stability, the four artificial complexes were separately subjected to 100 -ns MD simulations. The rmsd fluctuation of domain-peptide complex interface was monitored during the simulations. Here, the interfacial residues are defined as all peptide residues as well as those domain residues that are within $5 \AA$ distance from the peptide ligand. The rmsd fluctuation profile is a real-time measure of the complex stability during the simulations. As can be seen in Figure 2, the two complex systems of MAP3K SH3 domain with M1P and M2P peptides exhibit a stable dynamics trajectory over the whole simulations, while a larger thermal fluctuation can be observed for the two PI3K SH3-peptide complex systems.

Here, we visually examined the two MAP3K SH3-peptide complex systems at different stages of the simulations as well as the finally equilibrated structure after 100-ns simulations. It is shown in Figure 3A that the MAP3K SH3-M1P system can well kept in a standard SH3-peptide binding mode over the whole simulations, that is, the M1P peptide is folded into a PPII helix that can be tightly bound within the peptidebinding pocket (active pocket) of MAP3K SH3 domain. The total binding free energy $\Delta G_{\text {total }}$ of M1P peptide to MAP3K $\mathrm{SH} 3$ domain was predicted to $-6.7 \mathrm{kcal} / \mathrm{mol}$ by $\mathrm{MM} / \mathrm{PBSA}$ calculation and NMA analysis using the last 10-ns MD trajectory, indicating a favorable interaction in the domainpeptide recognition. The total binding free energy $\left(\Delta G_{\text {total }}\right.$ $=-5.7 \mathrm{kcal} / \mathrm{mol}$ ) can be decomposed into intermolecular interaction energy $\left(\Delta E_{\mathrm{int}}=-102.7 \mathrm{kcal} / \mathrm{mol}\right)$, desolvation effect $\left(\Delta G_{\mathrm{dslv}}=69.2 \mathrm{kcal} / \mathrm{mol}\right)$ and entropy penalty $(-T \Delta S$ $=27.8 \mathrm{kcal} / \mathrm{mol}$ ) (Table 1$)$. As can be seen, $\Delta E_{\text {int }}$ is very favorable for the domain-peptide binding, which, however, would be largely counteracted by the unfavorable $\Delta G_{\mathrm{dslv}}$ and $-T \Delta S$, finally resulting in a moderately favorable $\Delta G_{\text {total }}$. Fluorometric titration assay confirmed the calculated results (Figure 4, trace B). A micromolar affinity $\left(K_{\mathrm{d}}=96 \mu \mathrm{M}\right)$ can be obtained by titration curve fitting, suggesting a moderate interaction in MAP3K SH3-M1P recognition.

According to MD simulations, the M2P peptide cannot hold in the active pocket of MAP3K SH3 domain, although the dynamics trajectory of the complex system seems stable over the simulations (Figure 2, trace B). By visually examining the system equilibrated by MD simulations, it is found that the M2P peptide moves out of $\mathrm{SH} 3$ active pocket due to the simulations and finally fixes at a region nearby the pocket (Figure 3B). Binding free energy analysis revealed a modestly favorable interaction between the domain and peptide with $\Delta G_{\text {total }}=-2.5 \mathrm{kcal} / \mathrm{mol}$, which can be decomposed into $\Delta E_{\text {int }}=-76.8 \mathrm{kcal} / \mathrm{mol}, \Delta G_{\mathrm{dslv}}=53.2 \mathrm{kcal} / \mathrm{mol}$ and $-T \Delta S=27.8 \mathrm{kcal} / \mathrm{mol}$ (Table 1 ). The system was also determined to have a weak binding potency with dissociation constant $K_{\mathrm{d}}=157 \mu \mathrm{M}$ (Figure 4, trace A), suggesting an atypical encounter adduct between MAP3K SH3 domain and M2P peptide. It is worth noting that the M2P peptide contains a fluorescently active tryptophan residue that may influence the fluorescence spectroscopy assay. In

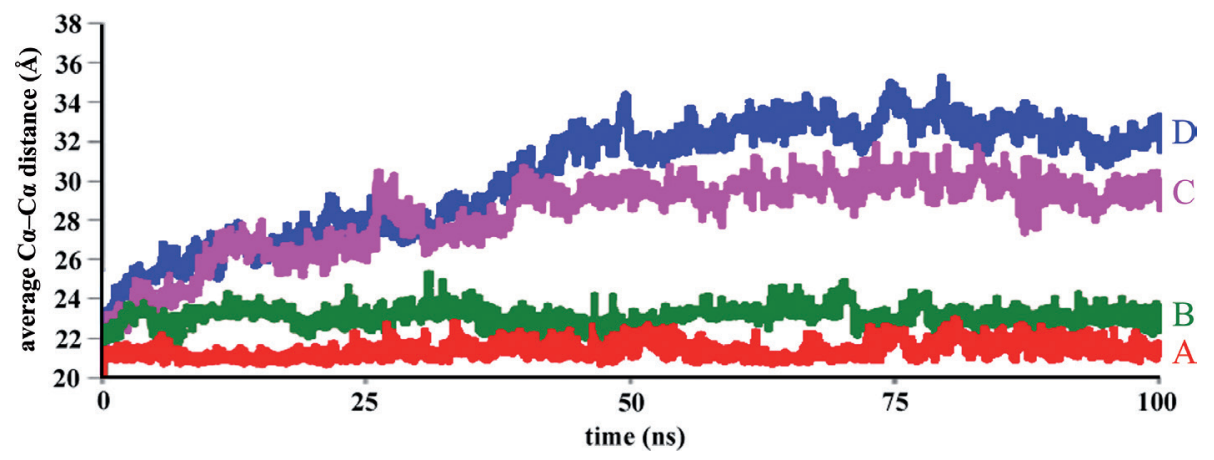

Figure 2. The rmsd fluctuation profiles of SH3-peptide complex interface over 100-ns MD simulations. A. MAP3K SH3-M1P system. B. MAP3K SH3-M2P system. C. PI3K SH3-M1P system. D. PI3K SH3-M2P system. 
A

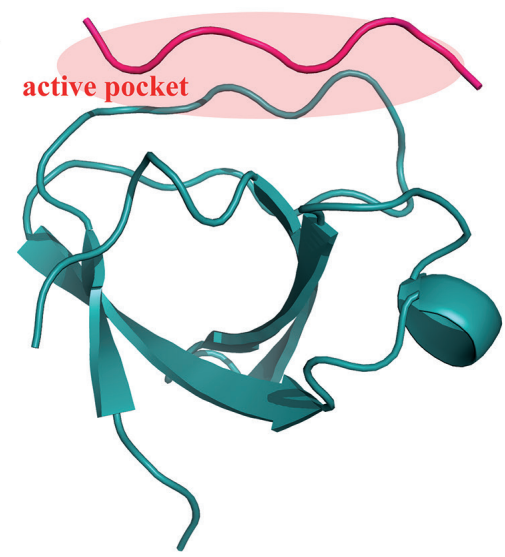

C

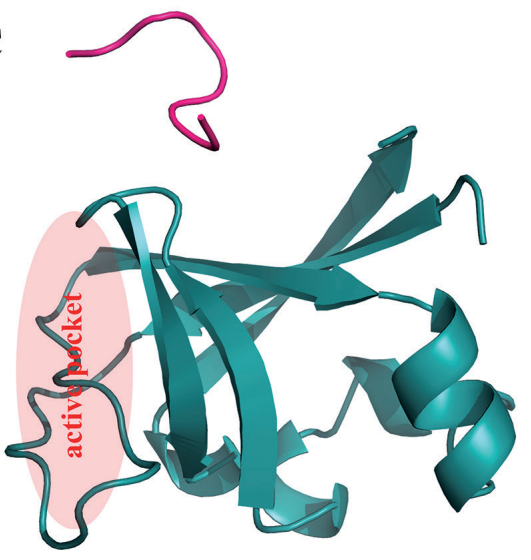

B
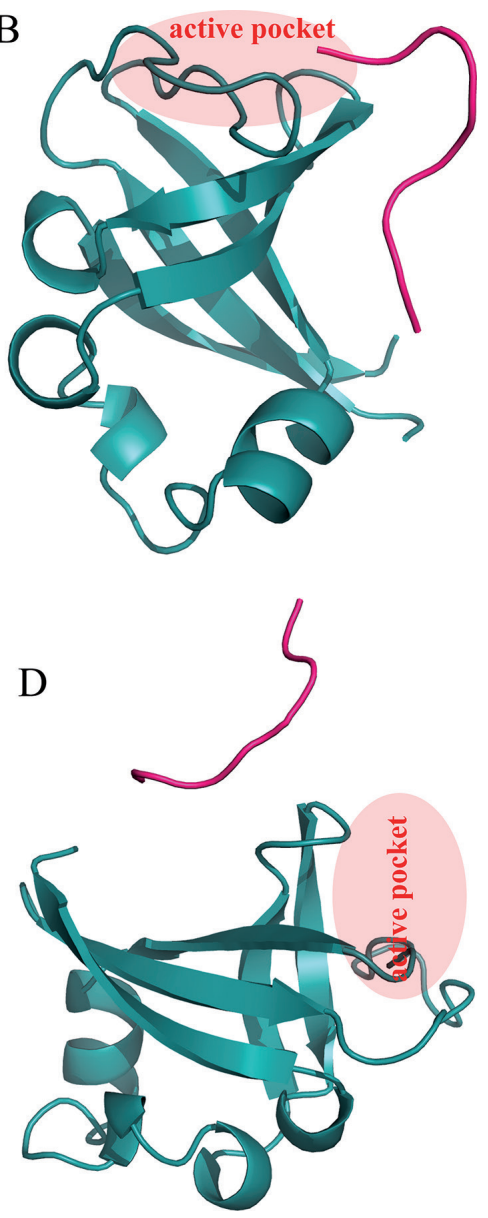

Figure 3. The equilibrated complex structures of $\mathrm{SH} 3$ domain and peptide ligand after 100 -ns MD simulations. A. MAP3K SH3-M1P system. B. MAP3K SH3-M2P system. C. PI3K SH3M1P system. D. PI3K SH3-M2P system. (Detailed description see in text). particular, the fluorescence intensity increase upon titration of the peptide to $\mathrm{SH} 3$ solution is almost linear (Figure 4A) that may be caused by adding M2P tryptophan to the monitored solution. However, the M2P peptide has been determined to exhibit the weakest binding capability $\left(K_{\mathrm{d}}\right.$ $=157 \mu \mathrm{M})$ to MAP3K SH3 domain in all the tested four peptides (i.e. M1P, M2P, M1Pm1 and M1Pm2). Therefore, even the current binding affinity is overestimated due to the presence of $\mathrm{M} 2 \mathrm{P}$ tryptophan residue (this means that the M2P peptide possesses a lower affinity or is a nonbinder), it would not influence our conclusion essentially.

On the other side, the peptides M1P and M2P cannot form stable complexes with $\mathrm{PI} 3 \mathrm{~K} \mathrm{SH} 3$ domain in terms of MD simulations. As can be seen in Figure 2, trace C and D,

Table 1. The calculated energetics data and experimental affinities for peptide binding to MAP3K and PI3K SH3 domains

\begin{tabular}{|c|c|c|c|c|c|}
\hline \multirow{2}{*}{ Domain-peptide system } & \multicolumn{4}{|c|}{ Energetics $(\mathrm{kcal} / \mathrm{mol})$} & \multirow{2}{*}{$K_{\mathrm{d}}^{e}(\mu \mathrm{M})$} \\
\hline & $\Delta E_{\text {int }}$ & $\Delta G_{\mathrm{dslv}}$ & $-T \Delta S$ & $\Delta G_{\text {total }}$ & \\
\hline MAP3K SH3-M1P & $-102.7 \pm 6.2$ & $69.2 \pm 4.3$ & $27.8 \pm 6.4$ & $-5.7 \pm 0.9$ & $96 \pm 7$ \\
\hline MAP3K SH3-M2P & $-76.8 \pm 3.8$ & $53.2 \pm 4.7$ & $21.1 \pm 4.8$ & $-2.5 \pm 0.4$ & $157 \pm 19$ \\
\hline PI3K SH3-M1P & $-26.4 \pm 2.4$ & $15.0 \pm 1.6$ & $9.7 \pm 2.6$ & $-1.7 \pm 0.2$ & - \\
\hline PI3K SH3-M2P & $-14.8 \pm 1.6$ & $7.8 \pm 0.9$ & $6.2 \pm 1.8$ & $-0.8 \pm 0.09$ & - \\
\hline MAP3K SH3-M1Pm1 & $-119.3 \pm 7.9$ & $80.4 \pm 5.6$ & $30.4 \pm 5.0$ & $-8.5 \pm 2.0$ & $17 \pm 1.2$ \\
\hline MAP3K SH3-M1Pm2 & $-108.6 \pm 5.4$ & $71.5 \pm 5.1$ & $31.0 \pm 5.7$ & $-6.1 \pm 1.5$ & $78 \pm 5$ \\
\hline
\end{tabular}

$\Delta E_{\text {int }}$, the interaction energy between domain and peptide; $\Delta G_{\text {dslv }}$ the desolvation free energy upon domain-peptide binding; $-T \Delta S$, the entropy penalty upon domain-peptide binding; $\Delta G_{\text {total }}$, the total free energy of domain-peptide binding; $K_{\mathrm{d}}$, the dissociation constant of domain-peptide binding. 


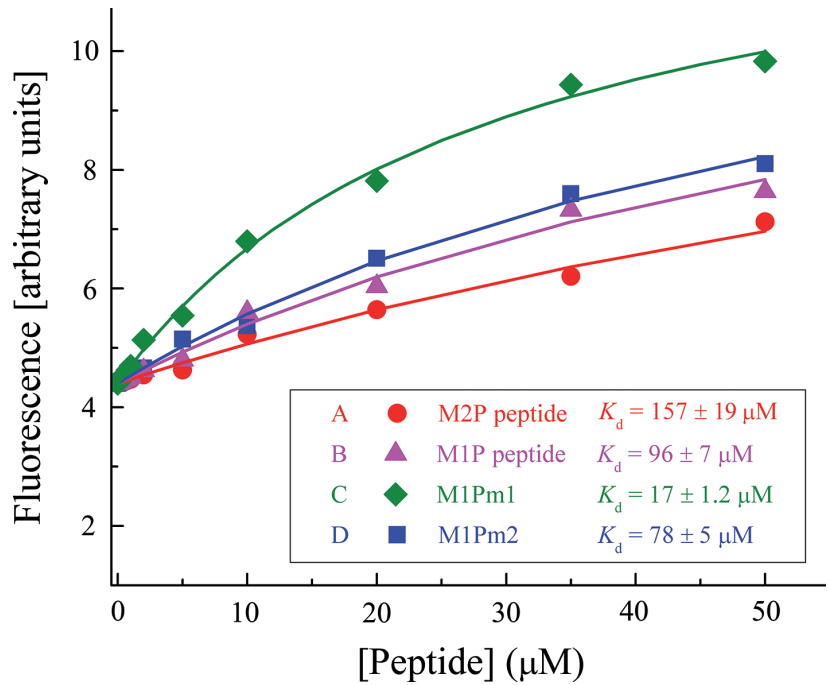

Figure 4. Titration of different peptides to the recombinant protein of MAP3K SH3 domain.

the rmsd changes gradually along the simulations, indicating that the two complex systems are unstable during the simulations and finally hold in an unfixed location far away from the active pocket. In addition, the peptide ligands cannot be structured that present in a random coiled conformation after the simulations (see Figure 3C and D). All these suggest that the PI3K SH3 domain may not be the native binding partner of M1P and M2P peptides. Binding free energy calculations can also support this notion, with resulting $\Delta G_{\text {total }}=-1.7$ and $-0.8 \mathrm{kcal} / \mathrm{mol}$ for the two peptides, respectively (Table 1).

Rational design of potent peptide ligands to target MAP3K SH3 domain

By combining in silico analysis and in vitro assay we demonstrated that the two hPTTG1 SH3-binding motifs have a moderate/modest affinity to MAP3K SH3 domain, suggesting that the Ser/Thr-specific protein kinase MAP3K can potentially phosphorylates hPTTG1 by recognizing and binding the motifs through its $\mathrm{SH} 3$ domain. MAP3K belongs to the family of mitogen-activated protein kinases (MAPKs); the kinase family is a key regulator of hPTTG1 signaling (Pei 2000; Vlotides et al. 2006; Chen et al. 2013) and has been showed to in vitro phosphorylate PTTG1 (Pei 2000). In the current study, it is found that the motif 1 peptide M1P of hPTTG1 can bind MAP3K SH3 domain in a standard mode

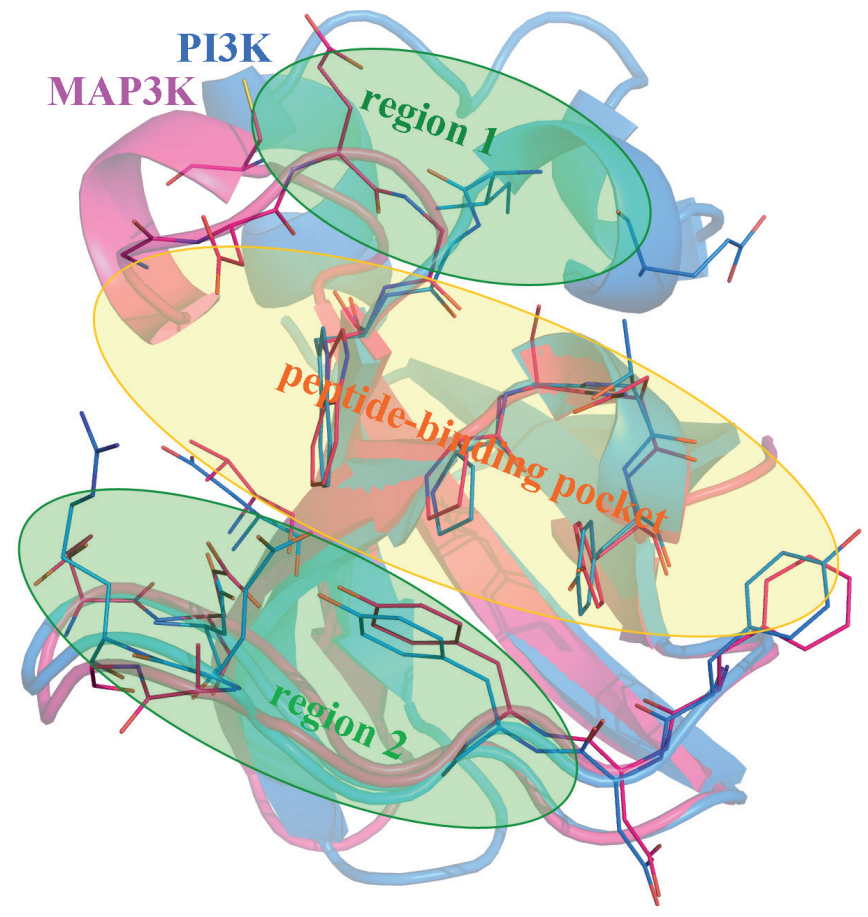

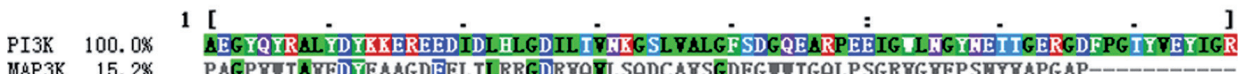

Figure 5. Sequence alignment and structural superposition between the SH3 domains of PI3K and MAP3K. (Detailed description see in text). 
(PPII helix) and exhibits a potent interaction with the domain $\left(K_{d}=96 \mu \mathrm{M}\right)$. Here, we attempted to modify the M1P peptide to derive mutant peptides with higher affinity for the domain. According to the modeled complex structure of MAP3K SH3 domain with M1P peptide, the positively charged Lys 168 residue at peptide C-terminus can form two salt bridges with domain residues Asp26 and Glu28. A number of backbone hydrogen bonds are observed between the domain and the core region ${ }^{163} \mathrm{PPSP}^{166}$ of the peptide, which confer stability and specificity to the complex system.

The sequence and structure similarity between the $\mathrm{SH} 3$ domains of MAP3K and PI3K were analyzed. As can be seen in Figure 5, the two domains have only a low sequence homology with identity $=15.2 \%$ derived from the sequence alignment using MView server (Brown et al. 1998). Structural superposition revealed that the two domains share a common folding model over global structural architecture; a conserved residue distribution pattern can be observed in their core peptide-binding pocket (Figure 5). However, there are two regions flanking to the pocket that exhibit low residue conservation over the two $\mathrm{SH} 3$ domains; the regions 1 and 2 are considerably and partially different between the two domains, respectively (Figure 5). Thus, the two regions may primarily contribute to peptide selectivity. By examining hydrophobic potential map on domain surface, it is evident that the peptide-binding pocket of $\mathrm{SH} 3$ domain can be divided into hydrophilic, hydrophobic and amphiphilic regions corresponding to the $\mathrm{N}$-terminus, central part and C-terminus of peptide ligand, respectively. The central part covers peptide core motif PXXP, which defines hydrophobic interactions and backbone hydrogen bonds with the domain. Considering that this part is critical for peptide binding, we herein did not modify it. The $\mathrm{N}$-terminus of peptide is very close to a negatively charged region (Asp55 and Glu56) of the domain, and we therefore considered mutating the nonpolar residue Leu161 to a positively charged residue Lys at the peptide $\mathrm{N}$-terminus to match the electrostatic complementarity between domain and peptide, resulting in the M1P mutant 1 KGPPSPVK (M1Pm1). In addition, the C-terminal Lys168 residue can form two salt bridges with the domain, which was thus fixed in the modification. However, the nonpolar residue Val167 adjacent to Lys168 seems to touch on the hydrophobic patch of domain surface, of which the side chain is relative small and cannot fill in the space between the residue and domain. Thus, we considered mutating the residue to a nonpolar, bulky and flexible amino acid Leu, resulting in the M1P mutant 2 LGPPSPLK (M1Pm2).

Subsequently, the complex structures of MAP3K SH3 domain with the two mutants were computationally modeled and then subjected to $100-n s$ MD simulations. As might be expected, the complexes were bound tightly and the mutant peptides were always kept in PPII structure state during the simulations, suggesting a dynamics stability of the two domain-mutant complexes. The total binding free energy $\Delta G_{\text {total }}$, as expected, were calculated to -8.5 and $-6.1 \mathrm{kcal} /$ mol for M1Pm1 and M1Pm2 peptides, respectively (Table 1), which are comparable to or better than that of wild-type M1P peptide $\left(\Delta G_{\text {total }}=-5.7 \mathrm{kcal} / \mathrm{mol}\right)$, indicating a theoretically satisfactory binding profile of the two mutants. To test the rational design, the two mutants were synthesized and their affinities to MAP3K SH3 domain were determined to $K_{\mathrm{d}}=17$ and $78 \mu \mathrm{M}$, respectively, by using fluorescence spectroscopy (Figure 4C and D), confirming a good consistence between the computational analysis and experimental assay. In particular, the affinity of M1Pm1 increases by 5.6 -fold relative to that of wild-type M1P $\left(K_{\mathrm{d}}=96 \mathrm{kcal} / \mathrm{mol}\right)$, which can thus be considered as a good candidate to develop peptide-like inhibitors that can competitively disrupt the native interaction between hPTTG1 and MAP3K.

Acknowledgements. This work was supported by the Changhai Hospital Second Military Medical University.

Conflict of interest. The authors declare no conflict of interest.

\section{References}

Berman H. M., Westbrook J., Feng Z., Gilliland G., Bhat T. N., Weissig H., Shindyalov I. N., Bourne P. E. (2000): The protein data bank. Nucl. Acids Res. 28, 235-242

http://dx.doi.org/10.1093/nar/28.1.235

Boelaert K., Yu R., Tannahill L. A., Stratford A. L., Khanim F. L., Eggo M. C., Moore J. S., Young L. S., Gittoes N. J., Franklyn J. A., Melmed S., McCabe C. J. (2004): PTTG's C-terminal PXXP motifs modulate critical cellular processes in vitro. J. Mol. Endocrinol. 33, 663-677

http://dx.doi.org/10.1677/jme.1.01606

Brown N. P., Leroy C., Sander C. (1998): MView: a web-compatible database search or multiple alignment viewer. Bioinformatics $14,380-381$

http://dx.doi.org/10.1093/bioinformatics/14.4.380

Case D. A., Cheatham T. E., Darden T., Gohlke H., Luo R., Merz K. M. Jr, Onufriev A., Simmerling C., Wang B., Woods R. J. (2005): The Amber biomolecular simulation programs. J. Comput. Chem. 26, 1668-1688 http://dx.doi.org/10.1002/jcc.20290

Chen P. Y., Yen J. H., Kao R. H., Chen J. H. (2013): Downregulation of the oncogene PTTG1 via the KLF6 tumor suppressor during induction of myeloid differentiation. PLoS One 8, e71282 http://dx.doi.org/10.1371/journal.pone.0071282

Darden T., York D., Pedersen L. (1993): Particle mesh Ewald: an $\mathrm{N}-\log (\mathrm{N})$ method for Ewald sums in large systems. Chem. Phys. 98, 10089-10092

http://dx.doi.org/10.1063/1.464397

Domínguez A., Ramos-Morales F., Romero F., Rios R. M., Dreyfus F., Tortolero M., Pintor-Toro J. A. (1998): hpttg, a human homologue of rat pttg, is overexpressed in hematopoietic neoplasms. 
Evidence for a transcriptional activation function of hPTTG. Oncogene 17, 2187-2193 http://dx.doi.org/10.1038/sj.onc.1202140

Duan Y., Wu C., Chowdhury S., Lee M. C., Xiong G., Zhang W., Yang R., Cieplak P., Luo R., Lee T., Caldwell J., Wang J., Kollman P. (2003): A point-charge force field for molecular mechanics simulations of proteins based on condensed-phase quantum mechanical calculations. J. Comput. Chem. 24, 1999-2012 http://dx.doi.org/10.1002/jcc.10349

Homeyer N., Gohlke H. (2012): Free energy calculations by the molecular mechanics Poisson-Boltzmann surface area method. Mol. Inf. 31, 114-122 http://dx.doi.org/10.1002/minf.201100135

Hou T., Chen K., McLaughlin W. A., Lu B., Wang W. (2006): Computational analysis and prediction of the binding motif and protein interacting partners of the Abl SH3 domain. PLoS Comput. Biol. 2, e1 http://dx.doi.org/10.1371/journal.pcbi.0020001

Jorgensen W. L., Chandrasekhar J., Madura J. D., Impey R. W., Klein M. L. (1983): Comparison of simple potential functions for simulating liquid water. J. Chem. Phys. 79, 926-935 http://dx.doi.org/10.1063/1.445869

Krieger E., Koraimann G., Vriend G. (2002): Increasing the precision of comparative models with YASARA NOVA - a selfparameterizing force field. Proteins 47, 393-402 http://dx.doi.org/10.1002/prot.10104

Krivov G. G., Shapovalov M. V., Dunbrack R. L. Jr. (2009): Improved prediction of protein side-chain conformations with SCWRL4. Proteins 77, 778-795 http://dx.doi.org/10.1002/prot.22488

Li H., Yin C., Zhang B., Sun Y., Shi L., Liu N., Liang S., Lu S., Liu Y., Zhang J., Li F., Li W., Liu F., Sun L., Qi Y. (2013): PTTG1 promotes migration and invasion of human non-small cell lung cancer cells and is modulated by miR-186. Carcinogenesis 34, 2145-2155 http://dx.doi.org/10.1093/carcin/bgt158

Moritsugu K., Njunda B. M., Smith J. C. (2010): Theory and normalmode analysis of change in protein vibrational dynamics on ligand binding. J. Phys. Chem. B 114, 1479-1485 http://dx.doi.org/10.1021/jp909677p

Pei L., Melmed S. (1997): Isolation and characterization of a pituitary tumor-transforming gene (PTTG). Mol. Endocrinol. 11, 433-441 http://dx.doi.org/10.1210/mend.11.4.9911

Pei L. (2000): Activation of mitogen-activated protein kinase cascade regulates pituitary tumor-transforming gene transactivation function. J. Biol. Chem. 275, 31191-31198 http://dx.doi.org/10.1074/jbc.M002451200

Pisabarro M. T., Serrano L. (1996): Rational design of specific highaffinity peptide ligands for the $\mathrm{SH} 3$ domain. Biochemistry 35 , 10634-10640 http://dx.doi.org/10.1021/bi960203t

Ryckaert J. P., Ciccotti G., Berendsen H. J. C. (1977): Numerical integration of the Cartesian equations of motion of a system with constraints: molecular dynamics of n-alkanes. J. Comput. Phys. 23, 327-341 http://dx.doi.org/10.1016/0021-9991(77)90098-5

Salehi F., Kovacs K., Scheithauer B. W., Lloyd R. V., Cusimano M. (2008): Pituitary tumor-transforming gene in endocrine and other neoplasms: a review and update. Endocr. Relat. Cancer $15,721-743$ http://dx.doi.org/10.1677/ERC-08-0012

Sánchez-Puig N., Veprintsev D. B., Fersht A. R. (2005): Human full-length Securin is a natively unfolded protein. Protein Sci. 14, 1410-1418 http://dx.doi.org/10.1110/ps.051368005

Schweimer K., Hoffmann S., Bauer F., Friedrich U., Kardinal C., Feller S. M., Biesinger B., Sticht H. (2002): Structural investigation of the binding of a herpesviral protein to the $\mathrm{SH} 3$ domain of tyrosine kinase Lck. Biochemistry 41, 5120-5130 http://dx.doi.org/10.1021/bi015986j

Tfelt-Hansen J., Kanuparthi D., Chattopadhyay N. (2006): The emerging role of pituitary tumor transforming gene in tumorigenesis. Clin. Med. Res. 4, 130-137 http://dx.doi.org/10.3121/cmr.4.2.130

Tong Y., Eigler T. (2009): Transcriptional targets for pituitary tumor-transforming gene-1. J. Mol. Endocrinol. 43, 179-185 http://dx.doi.org/10.1677/JME-08-0176

Unipro C. (2010): Ongoing and future developments at the Universal Protein Resource. Nucleic Acids Res. 39, D214-219

Vlotides G., Cruz-Soto M., Rubinek T., Eigler T., Auernhammer C. J., Melmed S. (2006): Mechanisms for growth factorinduced pituitary tumor transforming gene-1 expression in pituitary folliculostellate TtT/GF cells. Mol. Endocrinol. 20, 3321-3335 http://dx.doi.org/10.1210/me.2006-0280

Vlotides G., Eigler T., Melmed S. (2007): Pituitary tumor-transforming gene: physiology and implications for tumorigenesis. Endocr. Rev. 28, 165-186 http://dx.doi.org/10.1210/er.2006-0042

Yang C., Zhang S., He P., Wang C., Huang J., Zhou P. (2015): Selfbinding peptides: folding or binding? J. Chem. Inf. Model. 55, 329-342 http://dx.doi.org/10.1021/ci500522v

Yang C., Zhang S., Bai Z., Hou S., Wu D., Huang J., Zhou P. (2016): A two-step binding mechanism for the self-binding peptide recognition of target domains. Mol. Biosyst. 12, 1201-1213 http://dx.doi.org/10.1039/C5MB00800J

Zhang X., Horwitz G. A., Heaney A. P., Nakashima M., Prezant T. R., Bronstein M. D., Melmed S. (1999a): Pituitary tumor transforming gene (PTTG) expression in pituitary adenomas. J. Clin. Endocrinol. Metab. 84, 761-767 http://dx.doi.org/10.1210/jcem.84.2.5432

Zhang X., Horwitz G. A., Prezant T. R., Valentini A., Nakashima M., Bronstein M. D., Melmed S. (1999b): Structure, expression, and function of human pituitary tumor-transforming gene (PTTG). Mol. Endocrinol. 13, 156-166 http://dx.doi.org/10.1210/mend.13.1.0225

Received: May 5, 2016

Final version accepted: June 9, 2016

First published online: October 27, 2016 